\title{
LAS FORMAS FIJAS Y SUS MÁRGENES: SOBRE “ESTRUCTURAS DE SENTIMIENTO” DE RAYMOND WILLIAMS. UNA TRAYECTORIA
}

\author{
The fixed forms and its margins: \\ about "Structures of feeling" of Raymond Williams. A trajectory
}

Jorge Cáceres Riquelme*

Hugo Herrera Pardo **

\begin{abstract}
RESUMEN
En los últimos quince años, tanto en el contexto anglosajón como hispanohablante, ha resurgido un interés por el pensamiento de Raymond Williams (1921-1988), materializado en la reedición de sus principales textos teóricos -en el contexto hispanohablante algunos de ellos han sido publicados por primera vez- e inclusive de algunas de sus novelas. El propósito del siguiente artículo es perseguir y exponer el desarrollo histórico de uno de los conceptos capitales en la obra del crítico galés: "Estructuras de sentimiento". Concepto que desde su formulación inicial fue pensado como una exploración de las zonas excluidas por el paradigma racionalista -fundamentalmente los procesos formativos y formadores y las instancias de percepción emocionales y experienciales-, el cual ha hegemonizado, tanto en la estética como en la teoría cultural, una serie de conceptos que operan como totalidades ya formadas o "formas fijas", al decir de Williams.
\end{abstract}

Palabras clave: Raymond Williams, Estructuras de sentimiento, formas fijas, experiencia, teoría cultural.

\footnotetext{
* Facultad de Humanidades y Educación, Departamento de Humanidades, Licenciatura en Letras mención Literatura, Universidad Andres Bello. Viña del Mar, Chile. Correo electrónico: jorgecaceresr@gmail.com

** Facultad de Humanidades y Educación, Departamento de Humanidades, Licenciatura en Letras mención Literatura, Universidad Andres Bello. Viña del Mar, Chile. Correo electrónico: hugo.herrerap@gmail.com

Recibido el 23 de enero de 2013. Aceptado el 26 de Julio de 2013.
} 


\begin{abstract}
In the last fifteen years, both Anglo and Spanish-speaking context, interest in the thought of Raymond Williams (1921-1988) has re-emerged, being materialized in the reissue of his main theoretical texts -in the Spanish-speaking context some of them have been published for the first time- and even some of his novels. The purpose of this article is to pursue and to expose the historical development of one of the capital concepts in Williams's work: "Structures of feeling". Concept that since its initial formulation was understood as an exploration of the areas excluded by the rationalist paradigm fundamentally the former and the formative processes and the instances of emotional and experiential perceptions-, which has dominates, in both aesthetics and cultural theory, a series of concepts that operate as formed totalities or "fixed forms", according to Williams.

Keywords: Raymond Williams, Structures of feeling, fixed forms, experience, cultural theory.
\end{abstract}

\title{
INTRODUCCIÓN
}

Hay un pasaje del 18 Brumario de Luis Bonaparte de Karl Marx que pareciera ser uno de los hipotextos sobre los cuales Raymond Williams construyó su proyecto crítico. Es el pasaje en el que Marx se pregunta por los motivos que separan a legitimistas y orleanistas, "las dos grandes fracciones del partido del orden”, tras los eventos derivados del periodo 1848-1851:

"Lo que separaba, pues, a estas fracciones no era eso que llaman principios, eran sus condiciones materiales de vida, dos especies distintas de propiedad; era el viejo antagonismo entre la ciudad y el campo, la rivalidad entre el capital y la propiedad del suelo. Que, al mismo tiempo, había viejos recuerdos, enemistades personales, temores y esperanzas, prejuicios e ilusiones, simpatías y antipatías, convicciones y artículos de fe y principios que los mantenían unidos a una dinastía, ¿quién lo niega? Sobre las diversas formas de propiedad, sobre las condiciones sociales de existencia, se levanta toda una sobreestructura (en otras traducciones figura como superestructura) de sentimientos, ilusiones, modos de pensar y concepciones de vida diversos y plasmados de un modo peculiar. La clase entera los crea y los plasma sobre la base de sus condiciones materiales y de las relaciones sociales correspondientes. El individuo aislado, que los recibe por tradición y educación, podrá creer que son los motivos determinantes y el punto de partida de su conducta" (Marx, 1971: 50-51. Cursivas nuestras).

Este pasaje aparece en dos de los textos más relevantes de la obra de Williams, en los que el crítico galés relee la transición de va de Marx al marxismo en el marco de una tradición ligada a los estudios literarios. Nos referimos a los textos 
"Marxismo y cultura", incluido en Culture and Society (1958), y a su propuesta más exhaustiva y sistemática al respecto, Marxism and Literature (1977). Con todo, el énfasis de la utilización de las citas en ambos textos es distinto. Por un lado, en "Marxismo y cultura" Williams intenta reconocer la complejidad asignada por Marx a los procesos culturales, haciendo hincapié en que la "superestructura" posee distintos componentes, de los cuales una parte es "pura racionalización", en tanto que la otra parte se encuentra compuesta por lo afectivo, o en palabras del propio Marx, por "toda una superestructura de sentimientos, ilusiones, hábitos de pensamiento y concepciones de la vida". Mientras, en el capítulo "Base y superestructura", de Marxismo y literatura, la misma cita persigue dos objetivos distintos: en primer lugar, se sitúa en un contexto de cuestionamiento hacia el marxismo clásico, que consideraba como la clave del análisis cultural marxista la relación base determinante y superestructura determinada; en segundo lugar, le sirve a Williams para perseguir la genealogía del concepto de superestructura y sus diversos usos. Lo interesante de la utilización de la cita en este capítulo es que, a partir de ella, Williams deduce el sentido de superestructura como "formas de conciencia", las cuales son el principal objeto de su proyecto crítico ${ }^{1}$ y que por sobre todo se cristalizarían en su hipótesis cultural de "estructuras de sentimiento", la que justamente persigue el momento de emergencia de aquellas formas de conciencia.

De modo preciso, la intención que mantiene el presente artículo es ofrecer una trayectoria de la noción "Estructuras de sentimiento" en la obra de Williams². Seguimiento que fundaremos a partir de la revisión y el comentario de ciertos hitos textuales en los que Williams elabora una reflexión teórica y crítica de su "hipótesis cultural": Preface to Film (1954), The Long Revolution (1961), Drama from Ibsen to Brecht (1968), The Country and the city (1973) y Marxism and Literature (1977).

Creemos necesario este trabajo dado que, hasta hoy, no tenemos conocimiento de algún estudio publicado en español que se enfoque en la "hipótesis cultural" williamsiana. Existen, por cierto, artículos que la mencionan y que desarrollan reflexiones interesantes en torno a ella -imposible soslayarla cuando se habla del pensamiento o de los aportes de Raymond Williams-, sin

\footnotetext{
Tanto en Culture and Society como en The Long Revolution, Williams rastrea los desplazamientos semánticos de conceptualizaciones claves para entender el desarrollo de la cultura moderna, tales como "cultura", "clase", "arte", "democracia", etc.

2 Cabe aclarar que el concepto de "trayectoria" no lo entendemos como una sucesión progresiva sino como una suerte de reflexión sostenida en torno a su hipótesis cultural.
} 
embargo, en ninguno de ellos la noción de "estructuras de sentimiento" constituye el centro de atención ${ }^{3}$.

No obstante, más allá de una fundamentación disciplinar, también creemos necesario este trabajo porque el concepto de "estructuras de sentimiento" constituye un pilar del pensamiento williamsiano debido a que su intención es "describir la relación dinámica entre experiencia, conciencia y lenguaje como formalizada y formante en el arte, en las instituciones y en las tradiciones" (Cevasco, 2003: 159), y al hacerlo, esto es precisamente lo que nos interesa, se encuentra explorando las zonas excluidas -los márgenes- de aquellas "formas fijas" a decir de Williams, de los productos definidos, o en otras palabras, de las metáforas de totalidad, dominantes en el pensamiento crítico del siglo XX, tales como "Estructura", "Código", "Sistema”, entre otras, las que basadas en un doble recorte -a saber, su naturaleza de operación eminentemente racional, a la vez que asimiladas como producto ya formado en lugar de analizar su proceso formativo y formador- operan como acto de exclusión para otras formas sociales fundamentales, como la experiencia y los sentimientos, las cuales el concepto williamsiano tiene por objetivo rescatar para el análisis social. En ningún sentido esta última apreciación involucra un análisis parcializado de la totalidad cultural. Muy por el contrario, para Williams el análisis efectivo de los procesos y relaciones sociales involucra mucho más que la manipulación y relación de formas fijas; en este sentido, aquella totalidad se hace legítima únicamente cuando se integran en sus análisis los distintos elementos que la constituyen, en un continuum que comprenda el estudio de "todas las actividades y sus interrelaciones sin otorgar prioridad a ninguna que decidamos abstraer" (Williams, 2003a: 55). Como el mismo Williams aseguraba a comienzos de los sesenta en relación con el arte: éste se encuentra "allí, como una actividad, con la producción, el comercio, la política, la formación de familias" (Williams, 2003a: 55).

Por último, podríamos enmarcar el presente trabajo dentro de una tendencia surgida en los últimos ańos que ha reactivado la obra de Raymond Williams tanto en el contexto anglosajón, con la reedición en 2011 de textos tales como The Long Revolution, The Country and the City y la novela The volunteers (Woodhams, 2012), como en el contexto hispanohablante, a través de la reedición y la traducción -en

\footnotetext{
${ }_{3}^{3}$ Sólo por dar algunos ejemplos, podríamos mencionar los siguientes títulos: Sarlo, Beatriz. "Raymond Williams y Richard Hoggart: sobre cultura y sociedad", Punto de Vista 6 (1979): 9-18; Altamirano, Carlos. "Raymond Williams: proposiciones para una teoría social de la cultura", Punto de Vista 11 (1981): 20-23; Altamirano, Carlos. "Raymond Williams. Cultura. Sociología de la comunicación y del arte", Punto de Vista 19 (1983): 51; Altamirano, Carlos. "Raymond Williams, 1921 1988", Punto de Vista 33 (1988): 1-2; Sarlo, Beatriz. "Raymond Williams: una relectura", Punto de Vista 45 (1993): 12-15; Dalmaroni, Miguel. "Conflictos culturales: notas para leer a Raymond Williams", Punto de Vista 79 (2004): $42-46$.
} 
ciertos casos por primera vez- de algunos de sus más importantes textos, como Marxismo y literatura (2009), Palabras clave (2003) y La larga revolución (2003) (Dalmaroni, 2004).

\section{1. “ESTRUCTURAS DE SENTIMIENTO”: HISTORIA DE UN DES- PLIEGUE}

\subsection{Contra los dualismos: "estructuras de sentimiento" en Preface to Film (1954) y The Long Revolution (1961)}

Según John Higgins, el primer despliegue del concepto se halla en Preface to film (1954), texto escrito por Williams en conjunto con Michael Orrom. Para Higgins, desde sus inicios el término se plantea como un desafío abierto al marxismo ortodoxo en la medida en que ésta recaía constantemente, a la hora de los análisis literarios, en el dualismo reflectante de base y superestructura. En oposición a este esquema de razonamiento Williams propone la idea de "Estructura de sentimiento", la que entiende en este momento como un elemento "para lo que no hay una contraparte externa" y que "sólo puede ser percibido a través de la experiencia de la propia obra de arte" (Williams citado por Cevasco, 2003: 160). De esta manera, Williams lograría desmarcarse de la crítica marxista más tradicional, ya que mediante el concepto supera la concepción que apuntaba al determinismo de la base económica sobre las estructuras legales, religiosas, artísticas, y en general, sobre todas las formas de conciencia de una sociedad.

Para Williams, la experiencia viviente del mundo es inseparable de un todo complejo y es a partir de esta totalidad que el artista crea sus obras. De hecho, es principalmente en el arte donde "el efecto de la totalidad, la estructura de sentimiento, es expresada y encarnada" (Higgins, 1999: 41). Esto lleva al propio Williams a concluir que el concepto de "Estructura de sentimiento" tiende a convertirse en "una epistemología para conseguir la comprensión de toda la sociedad" (Higgins, 1999: 44) . Este primer acercamiento al concepto y su problemática deja en claro que el proyecto de crítica cultural pensado por Williams desde sus primeros textos pretendió ser en todo momento una historización e indagación de las respuestas tanto racionales como emocionales a las encrucijadas puestas por la modernización. De este modo, podría llegar a cuestionarse, y luego superarse, un análisis social sometido a formas fijas, es decir, totalidades

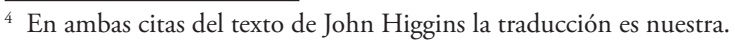


formadas antes que en formación. Con posterioridad, y de modo específico, este énfasis recaería en la sociedad inglesa desde fines del siglo XVIII. Lo conflictivo en términos teóricos y críticos es justificar, entonces, ese acercamiento emocional y sensorial a la cultura desde las obras literarias, fenómeno para el que precisamente postula el concepto de "estructuras de sentimiento", profundizando de esta manera un término anunciado ligeramente por Marx. Este trabajo de desarrollo y fundamentación adquirió consistencia con The Long Revolution (1961).

En este libro Williams dedica algunos apartados exclusivamente teóricos para justificar el fundamento de la relación entre comunicación y comunidad iniciado en Preface to Film. Desde sus primeros textos, Williams entendió que en donde mayormente se aprecia la pulsión de los cambios sociales es en el lenguaje. En este sentido, para llevar a cabo la tarea de fundamentar la naturaleza de la relación expresada más arriba, elige la práctica significante del concepto de "creativo", la que pesquisa desde las teorías imitativas de Platón y Aristóteles hasta las teorías creativas de Coleridge en adelante. A partir de tal práctica, Williams persigue la secularización progresiva de la palabra, dejando en evidencia que históricamente, en Occidente, la idea de creación estuvo ligada solo parcialmente a la idea de percepción. Puede sintetizarse el desarrollo histórico de esta problemática en base a las oposiciones “sujeto"/“objeto” o, en otras palabras, "arte”/“realidad”. Una teoría "imitativa” del arte concibió la actividad artística en términos de una descripción o reproducción corriente de la sociedad. Por su parte, la teoría "creativa" del arte entendía la relación entre éste y la realidad como una modificación subjetiva hecha por el artista. Inclusive un tercer tipo de teoría sobre el arte, que podría denominarse abstracto, ha entendido dicha relación como la expresión directa de una experiencia puramente estética. Para Williams, lo conflictivo de esta práctica significante de "creativo" se encuentra en tratar de comprenderlo como una oposición "arte"/"realidad" o "sujeto"/“objeto", la cual es en definitiva un falso significado, pues "la visión de la actividad humana que procuramos aprehender rechaza esa dualidad de sujeto y objeto: la conciencia es parte de la realidad y la realidad, parte de la conciencia, en el proceso general de nuestra organización viviente" (2003a: 36).

Es justamente debido a esto último que uno de los motivos de esta relegación experiencial del arte es su definición meramente abstracta, es decir, cuando pensamos en los fenómenos de forma separada, antitética. Enuncia Williams:

"La abstracción del arte ha sido su promoción o relegación a una zona de experiencia especial (emoción, belleza, fantasía, imaginación, inconsciente) a la que en la práctica 
nunca se limitó, ya que en realidad se extiende desde las actividades cotidianas más corrientes hasta crisis e intensidades excepcionales y utiliza una gama de medios, desde las palabras de la calle y las historias populares comunes hasta extraños sistemas de imágenes que, sin embargo, pudo convertir en propiedad de todos" (2003a: 50).

Es por ello que el crítico galés, haciendo un juego de palabras con una expresión tomada de Coleridge, en desmedro de este "conocimiento abstracto" de la cultura y el arte aboga por un "conocimiento sustancial" del mismo, que involucre el sentido de un proceso total. La intención que persigue Williams es una conceptualización de lo creativo que concierna a la totalidad de la experiencia sensorial ligada a la percepción, con el objetivo de vincularlo a "nuestra vida social corriente".

Los conceptos claves aquí son descripción y percepción. El primero de ellos Williams lo entiende como la organización, por parte del percipiente, de la actividad sensorial, la que, además, no debe reducirse a la mera transmisión sino que asimismo debe incluir la recepción y la respuesta. Por su parte, la percepción es comprendida como la interpretación, la descripción y la incorporación, a la organización del espectador, de la información transmitida por la obra, la cual es, a su vez y "en todos los niveles un ofrecimiento de experiencia, que puede ser aceptada, rechazada e ignorada" (Williams, 2003a: 42). En síntesis, el asunto de fondo es comprender la comunicación humana como un proceso social total, sin oposiciones o dualismos. Es precisamente esto a lo que Williams denomina "la larga revolución"; ${ }^{5}$ intentar por medio del análisis cultural una comprensión de la actividad creativa como todo un modo de vida, rompiendo así las perspectivas parcializadas sobre el arte -como lo hace la estética, que postula como una de sus características "la exclusión tácita de la comunicación como un hecho social" (Williams, 2003a: 42) - y proponiendo un análisis integrador que involucre la totalidad de la experiencia sensorial y social. En este contexto es que Williams entiende la noción de "Estructuras de sentimiento" como una instancia:

“... tan sólida y definida como lo sugiere el término "estructura”, pero [que] actúa en las partes más delicadas y menos tangibles de nuestra actividad. En cierto sentido, esa estructura de sentimiento es la cultura de un periodo: el resultado vital específico de

\footnotetext{
5 El título pone énfasis en el hecho de que se hace inadmisible pensar las "tres grandes revoluciones de nuestro tiempo", a saber, la revolución democrática, industrial y cultural, como procesos separados. "Todo nuestro modo de vida, desde la forma de nuestras comunidades hasta la organización y el contenido de la educación, y desde la estructura de la familia hasta el status del arte y el entretenimiento, es profundamente afectado por el progreso y la interacción de la democracia y la industria y la expansión de las comunicaciones" (Williams, 2003a: 13). Es necesario comprender el proceso en su conjunto y captarlo como una "larga revolución" si es que "pretendemos entender la crisis teórica, nuestra historia concreta o la realidad de la nuestra condición inmediata y los términos del cambio” (Williams, 2003a: 15).
} 
todos los elementos de la organización general. Y en este aspecto, las artes de un periodo, si consideramos que incluyen enfoques y tonos característicos de la argumentación, son de la mayor importancia” (Williams, 2003a: 57).

Para Williams, donde existen mayores probabilidades de ver expresadas las transformaciones experienciales que son objeto de las "Estructuras de sentimiento" es en las obras de arte, debido a que de ellas "se extrae naturalmente el sentido vital real, la profunda comunidad que hace posible la comunicación” (Williams, 2003a: 57). Williams se encuentra convencido de que lo que no puede perderse nunca de vista al analizar e interpretar los hechos artísticos es su capacidad de comunicación, ese particular entramado de percepciones y respuestas que vinculan las relaciones reales de una sociedad con sus formas y convenciones artísticas. La obra de arte es un hecho comunicable y comunicativo en la medida en que pasa a tornarse una experiencia comunitaria activa, por lo tanto, se vuelve una experiencia constitutiva y constituyente de la sociedad.

\subsection{Convenciones, formas y Estructuras de sentimiento en Drama from Ibsen to Brecht (1968) y The Country and the City (1973)}

En Drama from Ibsen to Brecht, Williams aborda el concepto de "Estructuras de sentimiento" en relación con la cuestión crítica de la forma dramática y lo define en su distinción respecto de un problema central para el teatro moderno, como lo es el de las "convenciones". Para el galés, una "convención” puede entenderse ya como un acuerdo tácito, ya como ciertos estándares aceptados que son inherentes al proceso de la producción teatral. Así, por ejemplo, el que en una obra de carácter naturalista las palabras y las acciones representadas se asemejen a las palabras y las acciones de la vida real no viene a ser más que un acuerdo -implícito o explícito, consciente o inconsciente- entre el autor, los actores y la audiencia, quienes consienten en que la obra opere de tal modo. En simples términos, de acuerdo a estas precisiones, una "convención” es una concesión, esto es, una aceptación de determinadas formas de representación. En este sentido, la "convención" puede comprenderse también como un método o una pieza técnica que facilita la puesta en escena. Ahora bien, los métodos y las técnicas varían, sin embargo, el público no siempre cambia a la par. Por ello, la puesta en práctica de una nueva forma artística debe sustentarse -dice Williams- en el acuerdo de 
dramaturgo, actores y espectadores; de ese modo, la novedad puede ser aceptada sin mayores fricciones. A fin de cuentas, se trata de una constante dependencia contractual entre los antedichos agentes; de allí que, en cualquier periodo, un alejamiento radical respecto de la tradición se vuelva una alternativa estéril al nivel de la comunicabilidad artística. En efecto - prosigue el autor-, en el teatro se requiere tanto de la tradición (la "convención" en cuanto acuerdo tácito) como de la experimentación (la "convención" en cuanto método dramático), pues la introducción de cualquier forma demasiado peculiar exige, con antelación, de una predisposición latente a aceptarla, de parte de toda la sociedad o al menos de parte de algunos de sus grupos.

Siguiendo estos lineamientos, colegimos que las "Estructuras de sentimiento" no se definen en contraposición absoluta a las "convenciones". Como hemos visto, el teatro se desarrolla a base de "convenciones"; puede emplear formas viejas o nuevas, tradicionales o experimentales, pero lo que no puede hacer es prescindir de ellas. De ahí que una "Estructura de sentimiento" no sea necesariamente el reverso de una "convención”. Por el contrario, en muchas ocasiones una "Estructura de sentimiento" resulta inseparable de una determinada "convención". Según Williams, una "Estructura de sentimiento" es en sí misma una "forma"; una "forma" particular al interior de una obra particular, que puede llegar a ser reconocida como una forma general y relacionada, por ende, a un periodo histórico-artístico. Lo distintivo de esta forma particular es que no posee un correlato externo a la obra misma:

“... en el análisis, es una experiencia común darse cuenta de que uno ha investigado la obra en términos de las partes separables, y siempre queda un elemento para el que no podemos encontrar un factor externo correspondiente. Es éste al que yo me refiero como estructura de sentimiento. Es tan firme y definido como una estructura, pero está basada en los más profundos y menos tangibles elementos de nuestra experiencia. Es un modo de responder a un mundo particular que en la práctica no es experimentado como un modo alternativo a otros, sino que es experimentado como el único modo posible. Sus medios, sus elementos, nos son proposiciones o técnicas; son sentimientos relacionados que se materializan. De la misma manera, es accesible a otros no a través de la argumentación lógica o las destrezas profesionales, por sí solos, sino por experiencia directa - una forma y un sentido, un sentimiento y un ritmo- en la obra de arte, la obra de teatro como un todo" (Williams, 1987: 18).

Como se observa, hay una continuidad entre Drama from Ibsen to Brechty las obras anteriormente señaladas, Preface to Film y The Long Revolution, en cuanto 
a las proposiciones en torno a "Estructuras de sentimiento". Esta continuidad encuentra su base en las ideas de que es en el arte donde mayormente pueden apreciarse las transformaciones de la experiencia aún no formalizada del todo y también en la inexistencia de un correlato externo para estas transformaciones, por lo cual resulta necesario explorar los márgenes de las totalidades formalizadas. Es decir, Williams, de modo progresivo, cae en la cuenta de lo fundamental que resulta ser cada vez más explícito en términos metodológicos para explicar su hipótesis cultural. Por ello es que propone en Drama from Ibsen to Brecht un análisis de la obra dramática enfocado, fundamentalmente, en las convergencias y divergencias entre "convenciones" y "Estructuras de sentimiento". Quizás el texto de Williams donde se aprecia desplegado con mayor potencial el concepto en cuestión sea The Country and the City (1973). En él desarrolla el cambio, al interior de la literatura inglesa y en el marco de las transformaciones que el capitalismo produjo en la sociedad de aquel país, en la "Estructura de sentimiento" que va desde la apreciación de la naturaleza como un orden recibido y natural (Jonson, Carew), pasando por un orden logrado pero precario (Marvell) y una enseñanza moral consciente: el mejoramiento de la naturaleza, la naturaleza como la obra del hombre antes que "un paraíso recibido o afortunado" (90), hasta las transformaciones más contemporáneas operadas por las relaciones capitalistas. Si en Drama from Ibsen to Brecht el énfasis metodológico estaba centrado en el análisis de las "convenciones", en The Country and the City el aspecto metodológico que más sobresale es el análisis de las "formas". Allí, por ejemplo, entrega una de las relaciones absolutamente relevantes entre Literatura y Sociedad: un género o forma, ya sea discursiva o literaria, nunca corresponde por completo a un solo sistema ideológico o social, sino que, más bien, acusa presiones y líneas de fuerza provenientes de la dinámica entre lo dominante, lo emergente y lo residual ${ }^{6}$, respondiendo a ellas a partir de propuestas y lógicas internas. Es el caso, por citar un ejemplo, de la formación dentro de la literatura inglesa de las formas poéticas asociadas a las casas solariegas. Williams crítica la continuidad desproblematizada que los estudios literarios han otorgado a este conjunto de poemas al incluirlos "en una misma tradición" (Williams, 2011: 88), lo que para el autor involucra una verdadera reificación de las casas mismas: como si la casa,

${ }^{6}$ En estrecha vinculación con lo hegemónico, Williams define lo dominante en el orden de aquellos rasgos que vienen a ser subordinantes dentro del proceso cultural total (2009: 165). Lo residual, por su parte, comprende aquello que "ha sido efectivamente formado en el pasado pero [que] todavía se halla en actividad en el proceso cultural" (2009: 167); lo residual puede estar incorporado a la cultura dominante tanto como desempeñar una función alternativa o de oposición frente a ella. Lo emergente, por otro lado, incluye también "elementos que son esencialmente alternativos u opositores" a la cultura dominante, no obstante, a diferencia de los elementos residuales, éstos no corresponden a formaciones sociales anteriores sino que "son creados de continuo" en el presente. 
y luego por derivación, sus ocupantes, fueran el signo evidente de un orden, aun cuando ese orden estaba siendo permanentemente reconstituido por la formación política y económica de una nueva aristocracia como, más tarde, lo sería por un nuevo capitalismo agrario (Williams, 2011: 89).

La exploración de Williams, en este sentido, avizora los alcances que las presiones sociales irradian sobre la literatura y la respuesta de ésta a través de una particular dinámica interna, dada, en este caso, por las transformaciones vividas por una forma específica en un determinado marco cultural. Esto se presenta como una de las principales potencialidades de la hipótesis cultural williamsiana, pues involucra en una relación simultánea de autonomía y dependencia a las formas literarias y a las experiencias sociales. Como señala Sarlo, "Williams encuentra las razones sociales que, presionando desde afuera de la literatura, pero desatando dentro de ella transformaciones formales, inducen cambios en las convenciones" (Sarlo, 2011: 18)

\subsection{La totalidad sentida: el concepto de "Estructuras de sentimiento" en Marxism and literature (1977)}

En la argumentación sostenida en torno a "Estructuras de sentimiento" en Marxism and literature (1977), Williams profundiza en los alcances metodológicos que abre el análisis de lo social viviente, lo activamente presencial, lo que en definitiva se encuentra "en solución" y no "precipitado", como expresa el autor. Williams continúa insistiendo en que el error básico de todo análisis marxista -y, de modo más amplio, cultural- es la reducción de lo social a formas fijas. Por lo general, los términos que son sometidos a análisis se presentan como formas sustanciales siempre definidas en pasado, como algo ya acabado, obviando que aquellos términos, en la práctica, constituyen formas singulares y en desarrollo. La notable ausencia de lo viviente y lo presencial en los análisis culturales ha dado cabida a la expresión de las tensiones sociales en términos de oposiciones que no hacen sino agudizar el error básico antes referido. El reto así planteado es, entonces, cómo dar cuenta de lo vivencial, lo que está ocurriendo y se encuentra en desarrollo y se constituye como una serie de procesos formadores y formativos antes que totalidades formadas. El crítico galés vuelve a insistir en este libro en

\footnotetext{
7 Una idea similar se encuentra en las propuestas de Jan Mukařovský cuando postula -comentado por René Jara- "que todo cambio en la estructura artística era provocado desde el exterior, sea directamente por el impacto inmediato de una transformación de la sociedad o indirectamente por el influjo del desarrollo de sectores paralelos de la cultura, como la ciencia, la economía o el mismo lenguaje. Sin embargo, el modo como el cambio es introducido y la forma a que da lugar dependen de los factores inherentes a la estructura artística” (Jara, 1972: 67).
} 
que "la alternativa real en relación con las formas fijas producidas y recibidas [...] Es un tipo de sentimiento y pensamiento efectivamente social y material, aunque cada uno de ellos en una fase embrionaria antes de convertirse en un intercambio plenamente articulado y definido" (Williams, 2009: 179). En el ámbito de la literatura, Williams venía definiendo a estos cambios (sentimientos específicos, ritmos específicos), desde sus trabajos anteriores, como "figuras semánticas", es decir, formas, notaciones o convenciones de cierta modalidad y cierta época.

Este cambio de objeto y perspectiva en el estudio de lo social empuja a preguntarse, en cada caso concreto de análisis, por interrogaciones abiertas, es decir, interrogantes históricas específicas tales como: ¿ha habido algún grupo que en este cambio cumpla una función de dominante o influyente?, ¿son estos cambios efectivamente resultados de una interacción más general? En palabras del propio Williams, esto se encuentra justificado en la medida en que "lo que estamos definiendo es una cualidad particular de la relación y la experiencia social, históricamente distinta de cualquiera otras cualidades particulares, que determinan el sentido de una generación o de un periodo" (Williams, 2009: 179). Ese hecho trae aparejado una importante consecuencia metodológica, la cual es que:

“[...] los cambios cualitativos específicos no son asumidos como epifenómenos de instituciones, formaciones y creencias modificadas, o simplemente como evidencia secundaria de relaciones económicas y sociales modificadas entre las clases y dentro de ellas. Al mismo tiempo son asumidos desde el principio como experiencia social antes que como experiencia "personal" o como el "pequeńo cambio" simplemente superficial o incidental de la sociedad" (Williams, 2009: 180).

De esta manera, para Williams se constituyen en sociales de dos modos que escapan a la comprensión reductiva de lo social, considerada tanto institucional como formalmente. En primer lugar, porque se articulan como cambios de presencia, es decir, como cambios que, en cuanto están siendo vividos, siempre se observan como elementos en tránsito. En segundo lugar, porque, aun en sus etapas más germinales, estas "figuras semánticas” no necesitan "esperar una definición, una clasificación o una racionalización antes de ejercer presiones palpables y de establecer límites efectivos sobre la experiencia y sobre la acción" (Williams, 2009: 180). Y, por último, precisamente porque esta forma de análisis propuesta por Williams escapa de versiones mayormente institucionales y formales de lo social es que asume deliberadamente la denominación de "Estructuras de sentimiento", 
para así escabullirse de conceptos de sentido ya formados como "ideología” o “concepción de mundo". Aunque esto no signifique que se trate solamente:

"[...] de que debamos ir más allá de las creencias sistemáticas y formalmente sostenidas, aunque por supuesto siempre debamos incluirlas. Se trata de que estamos interesados en los significados y valores tal como son vividos y sentidos activamente; y las relaciones existentes entre ellos y las creencias sistemáticas o formales, en la práctica son variables (incluso históricamente variables) en una escala que va desde un asentimiento formal con una disensión privada hasta la interacción más matizada existente entre las creencias seleccionadas e interpretadas y las experiencias efectuadas y justificadas" (Williams, 2009: 180).

Por el lado de la elección deliberada de "estructura", el énfasis recae precisamente en presentar el análisis como una serie de relaciones internas y específicas -y a la vez entrelazadas y en tensión- "del pensamiento tal como es sentido y del sentimiento tal como es pensado; una conciencia práctica de tipo presente dentro de una continuidad viviente e interrelacionada" (Williams, 2009: 180). En otras palabras, esta perspectiva coloca la atención sobre la relación y lo relacionado, para dar paso a interrogantes históricas específicas ${ }^{8}$.

Debido a la justificación de esta perspectiva metodológica es que el autor caracteriza a "estructuras de sentimiento" como una "hipótesis cultural", la cual se encuentra "derivada de los intentos por comprender tales elementos y sus conexiones en una generación o en un periodo, con permanente necesidad de retornar, interactivamente, a tal evidencia” (Williams, 2009: 181). Lo cual supone que aunque inicialmente "es menos simple que las hipótesis sobre lo social más formalmente estructuradas", se presenta como "más adecuada al rango real de evidencia cultural: es históricamente cierta, pero aún lo es más (donde más importa) en nuestro presente proceso cultural" (Williams, 2009: 181-182). Es de este modo que:

"La hipótesis tiene una especial relevancia con respecto al arte y la literatura, donde el verdadero contenido social está en un significativo número de casos de este presente y de tipo afectivo, que no pueden ser reducidos sin desmedro a sistemas de creencias, instituciones o relaciones generales explícitas, aunque pueden incluirlos a todos como elementos vividos y experimentados, con o sin tensión, del mismo modo que evidentemente también incluyen elementos de la experiencia social o material (física o natural) que pueden situarse más allá de, o hallarse descubierta o imperfectamente cubierta por los elementos sistemáticos reconocibles en cualquier parte. En el arte, la presencia inequívoca de ciertos elementos que no están cubiertos por (aunque de algún

\footnotetext{
8 "Por lo tanto, es una estructura específica de eslabonamientos particulares, acentuaciones y supresiones particulares y, en lo que son habitualmente sus formas más reconocibles, profundos puntos de partida y conclusiones particulares" (Williams, 2009: 183).
} 
modo pueden ser reducidos a) otros sistemas formales, constituye la verdadera fuente de las categorías especializadas de "lo estético", "las artes" y la "literatura imaginativa" (Williams, 2009: 182).

Las "figuras semánticas", objeto de las "Estructuras de sentimiento", son, por tanto, índices de procesos formadores y formativos, por lo que constituyen elementos consustanciales del proceso social material, y justamente por ello no pueden ser desatendidas de ningún análisis social que intente investigar la manera en que determinados momentos o periodos históricos son experimentados y vividos en su desarrollo, con todas las contradicciones y conflictos que ello implica.

Precisamente, en relación a esto último señalado es que surgen los grandes cuestionamientos teóricos que los críticos de Williams dejan caer sobre "Estructuras de sentimiento". Nos referimos a los conceptos de "Experiencia" y "Vida", que, según los entienden los entrevistadores de Williams en Politics and letters (1979) -Perry Anderson, Anthony Barnett y Francis Mulhern-, son nociones que implicarían la vuelta a un planteamiento idealista y conservador (como las propuestas de Leavis -el English-, en las que el mismo Williams fue formado en Cambridge). Los entrevistadores enjuician la desideologización que "Estructuras de sentimiento" propondría, desconociendo la mediación de la ideología en la comprensión de la realidad. Por su parte, Williams desmiente ese acercamiento inmediato y desproblematizado a la realidad que sus entrevistadores le endilgan. Como él mismo expone en la primera parte de The Long Revolution, no existe una manera natural de percibir la realidad, sino que "la realidad, tal como la experimentamos, es en este sentido, una creación humana; que toda nuestra experiencia es una versión humana del mundo que habitamos" (Williams, 2003a: 32).

En definitiva, la divergencia entre los entrevistadores y Williams en torno al concepto de experiencia puede explicarse recurriendo a los conceptos acuñados por Brian McHale: dominante epistemológica y dominante ontológica9. Los entrevistadores tienen una concepción epistemológica de la experiencia, como un proceso racional, mientras que el hincapié de Williams a lo largo de su vasta obra es argumentar en contra de esa reificación de la racionalidad, arguyendo que la experiencia es una actividad que involucra, además del componente racional, lo

\footnotetext{
9 A partir de planteamientos de Iuri Tinianov -la Dominante- y Richard Rorty -lo epistemológico versus lo ontológico-, McHale entiende a la "Dominante epistemológica" como un cuestionamiento sobre el conocimiento y las formas de conocer, mientras que "Dominante ontológica" la entiende como un cuestionamiento a la naturaleza y las reglas que gobiernan una determinada entidad (McHale, 1991: 9-10).
} 
sentimental ${ }^{10}$. Una segunda diferencia que se observa tiene que ver con el hecho de que, para Williams, las reglas o modelos a través de los cuales experimentamos la realidad pueden ser modificados por el individuo, posibilidad que el concepto marxista de ideología no permite vislumbrar tan fácilmente. Esta proposición entrega un nuevo fundamento al uso que Williams efectúa de la cita contenida en El 18 Brumario de Luis Bonaparte de Karl Marx, en donde el pensador alemán enfatizaba que la realidad está conformada por elementos tanto racionales como afectivos, por "toda una superestructura de sentimientos, ilusiones, hábitos de pensamiento y concepciones de la vida”.

En síntesis, "Estructuras de sentimiento" es la alternativa propuesta por Williams en contra de los análisis sociales y culturales basados en totalidades, es decir, formas fijas producidas y recibidas. La intención central del concepto es rescatar la experiencia social en proceso desde un análisis consecuentemente efectivo, lo que involucra colocar el foco de atención tanto en la interpretación recibida como en la experiencia práctica, con el objetivo de develar un tipo de pensamiento y sentimiento social y material tal como son vividos y experimentados, presentados así como síntomas de una instancia de germinación, esto es, antes de ser articulados de modo pleno.

En otras palabras, su objetivo es explorar las tensiones entre elementos constitutivos y constituyentes del proceso social material en el marco de relaciones reales. Debido a esto es que a lo largo de su postulación Williams enfatiza el término estructura, ya que las relaciones entre estos elementos superan en demasía el análisis que los reduce a intercambios sistemáticos entre unidades fijas, el término "estructura", en la acepción de Williams, apunta a que los proceso no solo tienen lugar entre, sino que también dentro de la relación y lo relacionado; "Estructura" como una serie de relaciones internas específicas, y a la vez entrelazadas y en tensión. Esta serie de relaciones en el análisis williamsiano la constituyen las "figuras semánticas", "formas" y "convenciones" que se presentan así como elementos inalienables del proceso social material.

\footnotetext{
${ }_{10}$ Williams retomaría la polémica en torno al concepto de "experiencia" en la versión ampliada de Keywords (1983). Allí, donde incluye una definición del término, distingue entre "experiencia pasada" y "experiencia presente". La primera la define como el "conocimiento reunido sobre los acontecimientos pasados, ya sea mediante la observación consciente o por la consideración y reflexión"; la segunda, en tanto, la comprende como "el tipo más pleno, abierto y activo de conciencia, y [que] además del pensamiento incluye el sentimiento" (Williams, 2003b: 138).
} 


\section{CONCLUSIONES}

Para Raymond Williams -concluimos a partir de nuestras lecturas-, hombres y mujeres no son entes pasivos, tablas rasas sobre las cuales escribir o meros productos de determinadas ideologías o concepciones de mundo. Muy por el contrario, las sociedades se hallan constituidas por sujetos que operan como receptores activos de significados, valores o creencias ya formalizados; sujetos cuya experiencia de la totalidad social y material presente es dinámica y cuya actividad puede, por lo mismo, tanto aceptar como contrariar aquella totalidad. De ahí que en sus análisis culturales Williams cuestione los dualismos de base-superestructura, sujeto-objeto y arte-realidad; para el galés, todas estas entidades son indisociables en tanto forman parte de un mismo proceso, esto es, en tanto unas y otras se limitan y se presionan mutuamente.

Esta determinación bilateral -por llamarla de algún modo-que Williams adopta para estudiar lo social se hace muy patente en su propuesta de "estructuras de sentimiento", ya que mediante esta "hipótesis cultural" pretende identificar la emergencia de una forma alternativa ${ }^{11}$ de experimentar (de sentir y de pensar) la totalidad social. Se trata de una vivencia de lo social que traza relaciones con los valores sistemáticos y formales, pero que también los tensiona. Por ello la definición de esta experiencia en proceso o en solución -de este sentimiento pensado y de este pensamiento sentido- como una "estructura"; y por ello, también -según Williams-, que donde mejor pueda apreciarse tal experiencia sea en el arte, pues en él se manifiestan elementos afectivos para los cuales no es dable encontrar contraparte externa.

En el relato de una visita a Raymond Williams durante el año 1981, que sirve de cierre para el prólogo a la edición en español de El campo y la ciudad, Beatriz Sarlo le pregunta el porqué del abandono, en sus últimos trabajos, de la noción de "estructuras del sentir". A lo que Williams responde que: “...se sentía aliviado de haber podido dejarla de lado como fórmula, que de todas maneras eso no iba a modificar su percepción de los procesos culturales y, en cambio, le ahorraba cantidad de pendencias teóricas y explicaciones que no tenía ganas de repetir" (2001: 21).

\footnotetext{
11 Estamos tentados de escribir "una forma nueva", pero nos contiene la definición de "nuevo" -en la terminología williamsiana- como actualización de lo dominante (Williams, 2009: 169). Por eso es que, aun cuando puede no ser el vocablo más preciso, optamos por hablar de "una forma alternativa", intentando mantener con ello el sentido de otredad y de oposición que expresa nuestro autor al referirse a las estructuras de sentimiento.
} 
Llama la atención la presencia de la palabra "fórmula" en un contexto de "pendencias teóricas". Y es que parece coherente el abandono de Raymond Williams del concepto en cuestión en una época en que la teoría y la metodología seguían funcionando como una relevante "tradición selectiva", comprendida como una serie de discursos jerarquizantes, hegemónicos, esencialistas. Parece coherente porque, precisamente, el concepto de "estructuras de sentimiento" invita a considerar alternativamente el discurso de la teoría y la metodología, en tanto busca ser una noción que no se preocupa por entregar tipologías ni modelos de análisis concretos, sino que por el contrario, su énfasis es siempre trabajar con interrogantes históricos abiertos, que en cada caso, faciliten la profundización de la coyuntura particular, localizada, y el rol de la subjetividad en ella. Y en ningún sentido proporcionar una teoría-metodología que entregue una fórmula de análisis que confunda a ésta con prescripción, y que desemboque, de esta manera, en una especie de nuevo formalismo. Unas palabras de Stuart Hall -claramente en una relación que huye de la casualidad-ayudan a completar esta percepción sobre el discurso de la teoría y sus expectativas de desarrollo posterior: "la única teoría que vale la pena tener es aquella con la que uno tiene que luchar, no aquella de la que uno habla con una fluidez profunda" (Hall, 2010: 54). Y también: "No la teoría como una voluntad de verdad, sino la teoría como un conjunto de conocimientos localizados, coyunturales, que deben debatirse de forma dialógica" (Hall, 2010: 63). Es específicamente esta la interpelación que "estructuras de sentimiento" realiza al sentido y usos de la teoría. Extender la noción a una fórmula o modelo habría sido una contradicción raigal para su argumentación sostenida por décadas. El abandono de ella, sin que eso involucre un cambio en su percepción del proceso cultural, aparece así como una opción más lógica.

Rescatando la definición que entrega Paul de Man sobre la teoría como una "reflexión controlada sobre la formación del método" (1990: 12), "Estructuras de sentimiento" pareciera inscribirse como la particular respuesta subversiva de Raymond Williams sobre aquella tradición selectiva. Es una respuesta particular y subversiva, porque, como intenta establecer Edward Said en su ensayo "Teoría ambulante", la lectura más relevante que realiza Williams sobre el desarrollo de la teoría es que en cada caso nunca deben dejar de auscultarse las limitaciones de ésta, ya que "un avance metodológico puede convertirse en una trampa si 
se utiliza de forma acrítica, repetitiva, ilimitada" (Said, 2004: 319) ${ }^{12}$. De este reconocimiento se desprende el valor de conciencia crítica en "Estructuras de sentimiento", porque si la labor de la teoría ha sido dar cuenta de un fenómeno ya sistematizado, lo que pretende develar la noción de Williams es precisamente de lo que todavía permanece como alternativo, emergente y aún no ha sido articulado sistemáticamente por el proceso social. Podemos decir, entonces, que es a partir de los intersticios, los puntos ciegos existentes entre un orden social dominante y sus alternativas es que se ubica la preocupación teórica de una noción como "estructuras de sentimiento". En este sentido, la categoría de "Estructuras de sentimiento" se presenta como la particular elaboración teórica de Williams con respecto a su visión de los sistemas sociales hegemónicos”... ningún modo de producción y por lo tanto ningún orden social dominante ni ninguna cultura dominante jamás en realidad incluye o agota toda la práctica humana, toda la energía humana y toda la intención humana” (2009: 171).

\section{REFERENCIAS}

Altamirano, Carlos, "Raymond Williams: proposiciones para una teoría social de la cultura", Punto de Vista 11 (1981): 20-23.

--------. "Raymond Williams. Cultura. Sociología de la comunicación y del arte”, Punto de Vista 19 (1983): 51.

. "Raymond Williams, 1921-1988”, Punto de Vista 33 (1988): 1-2.

Cevasco, María Elisa. Para leer a Raymond Williams, Universidad Nacional de Quilmes ediciones, Buenos Aires, 2003.

Dalmaroni, Miguel. "Conflictos culturales: notas para leer a Raymond Williams", Punto de Vista 79 (2004): 42-46.

\footnotetext{
12 A propósito de las últimas ideas discutidas, no deja de ser sugerente a este respecto la postura adoptada por Jan Mukařovskỳ. En su artículo "El Estructuralismo en la Estética y en la Ciencia Literaria", asume que sus postulados prefiere nominalizarlos como "posición", en lugar de "teoría" y "método", porque están últimas denotan un "conjunto definido de conocimientos y un conjunto definido e invariable de reglas de trabajo"(2000: 271). El "Estructuralismo", según Mukařovskỷ lo concebía, no es ni lo uno ni lo otro, sino más bien, "una postura epistemológica, de la cual se derivan determinadas reglas de trabajo y determinados conocimientos, pero que existe independientemente de ellos y es por tanto capaz de evolucionar en ambos aspectos" (2000: 271).
} 
De Man, Paul. La resistencia a la teoría. Madrid: Visor, 1990.

Hall, Stuart. Sin garantías. Trayectorias y problemáticas en Estudios Culturales. Popayán: Envión, 2010.

Higgins, John. Raymond Williams: literature, marxism and cultural materialism. New York: Routledge, 1999.

Jara, Rene. "La escuela de Praga y la teoría literaria", Problemas de Literatura 2 (1972): 65-71. Disponible en: <http://bagubra.ucv.cl/PDF/problemas-deliteratura-2.pdf>. [Consultado: el 5 de diciembre de 2012].

McHale, Brian. Postmodernism fiction. London and New York: Routledge, 1991.

Marx, Karl. 18 Brumario de Luis Bonaparte. Buenos Aires: Ariel, 1972.

Said, Edward. El mundo, el texto y el crítico. Buenos Aires: Debate, 2004.

Sarlo, Beatriz. "Raymond Williams y Richard Hoggart: sobre cultura y sociedad", Punto de Vista 6 (1979): 9-18.

."Raymond Williams: una relectura”, Punto de Vista 45 (1993): 12-15.

-_-_-_-_---.-. "Raymond Williams: del campo a la ciudad", Prólogo a R. Williams. El campo y la ciudad. Buenos Aires: Paidós, 2011.

Williams, Raymond. Drama from Ibsen to Brecht. London: The Hogarth Press, 1987.

------------. La larga revolución. Buenos Aires: Ediciones Nueva Visión, 2003a. Palabras clave. Un vocabulario de la cultura y la sociedad. Buenos Aires: Ediciones Nueva Visión, Buenos Aires, 2003b.

Marxismo y Literatura. Buenos Aires: Las Cuarenta, 2009.

---------.-. El campo y la ciudad. Buenos Aires: Paidós, 2011.

Woodhams, Steve. "Reading Raymond Williams in 2012". Disponible en: <http://www.raymondwilliamsfoundation.org.uk/pdf/Reading\%20 Raymond\%20Williams\%20in\%202012.pdf>. [Consultado: el 30 de agosto de 2012]. 